\title{
Comparative Study on Polarization Spectral Parameters of Fruit in Southern Xinjiang Based on Roujean Model
}

\author{
Jiayi Xu1,3, Xue Huang2,3, Huaping Luo',3, Yuting Suo',3, Feng Gao1,3 \\ ${ }^{1}$ College of Mechanical and Electrical Engineering, Tarim University, Alar, Xinjiang, China \\ ${ }^{2}$ College of Plant Science, Tarim University, Alar, Xinjiang, China \\ ${ }^{3}$ The Key Laboratory of Colleges and Universities under the Department of Education of Xinjiang Uygur Autonomous Region, \\ Alar, Xinjiang, China \\ Email:1056939585@qq.com
}

How to cite this paper: Xu, J.Y., Luo, H.P., Suo, Y.T., Huang, X. and Gao, F. (2021) Comparative Study on Polarization Spectral Parameters of Fruit in Southern Xinjiang Based on Roujean Model. American Journal of Analytical Chemistry, 12, 59-73. https://doi.org/10.4236/ajac.2021.123005

Received: February 18, 2021

Accepted: March 22, 2021

Published: March 25, 2021

Copyright $\odot 2021$ by author(s) and Scientific Research Publishing Inc. This work is licensed under the Creative Commons Attribution International License (CC BY 4.0).

http://creativecommons.org/licenses/by/4.0/

\begin{abstract}
Based on the study of phase angle and wavelength in pBRDF (Polarized bidirectional reflectance distribution function), roujean model was proposed to describe Orient (Polarization phase angle) quantitatively. The Roujean model was used to quantitatively describe different fruits intensity components $\left(F_{00}\right)$ and polarization phase angle (Orient), and the simulation results were analyzed and compared using statistical analysis and comparison methods to realize the prediction from the regular model to the outdoor fruit tree canopy to the canopy of outdoor fruit tree canopy random distribution. The experimental results showed that: 1) when the phase angle of jujube was $52.19^{\circ}$, $66.51^{\circ}$ and $88.26^{\circ}$, the $R^{2}$ and average errors of $F_{00}$ parameters described by Roujean model are $0.9982,0.9963,0.9912$ and $3.80 \%, 4.17 \%, 6.40 \%$, respectively; and the $R^{2}$ and average error of Orient parameters described by Roujean model are $0.9056,0.9223,0.9260$ and $6.23 \%, 3.32 \%, 8.05 \%$, respectively; It can be seen that roujean model can quantitatively describe the Orient parameter of jujube; 2) When the phase angle of apricot was $70.99^{\circ}, 71.28^{\circ}$ and $67.91^{\circ}$, the $R^{2}$ and average errors of $F_{00}$ parameters described by Roujean model is $0.9862,0.9823,0.9792$ and $3.40 \%, 4.82 \%, 5.19 \%$, respectively; And the $\mathrm{R}^{2}$ and average error of Orient parameters described by Roujean model are $0.9382,0.8947,0.8849$ and $7.19 \%, 9.28 \%, 9.47 \%$, respectively. Roujean model can also quantitatively describe the Orient parameter of white apricot. In summary, the Roujean model can provide a good quantitative description of $f_{00}$ and a good quantitative description of Orient, which in turn can predict the pBRDF parameter for more fruits with different incidence and detection directions. It can correct the influence of angle factor in the nondestructive testing of outdoor fruits.
\end{abstract}




\section{Keywords}

Bidirectional Reflectivity Distribution Function, Degree of Linear Polarization, Intensity Component, Roujean Model, Phase Angle

\section{Introduction}

Roujean model is a kind of surface bidirectional reflectance model, which is utilized to correct the influence of surface bidirectional reflectance in the time series of satellite observations. The model follows the semi empirical method and is appropriate for all kinds of heterogeneous new surfaces. In the field of materials, Yang Peiyan and others applied it to study the bi-directional properties of PTFE sheet diluent, aiming at reducing the temperature of the object under direct sunlight without refrigeration and consuming power [1]; Inoue and others used it to evaluate the surface morphology of paper, aiming at explaining the glossiness phenomenon of paper [2]. In the industrial field, Luongo et al. [3] used it in the research of three-dimensional printing technology to provide a tool for users to estimate the reflectivity of printer control surface; Zhang Yingluo et al. [4] used it in the design of underwater laser detection system. In the military field, Melvin et al. [5] applied it to remote detection of mines and improvised explosive devices. In the aviation field, Yeom et al. [6] used it to detect thin clouds on the ground, which improved the efficiency of determining thin cloud pixels; Farhad et al. [7] used it to develop a new cross calibration technology for satellite sensors, so as to improve the data coordination between them. In the marine field, aval et al. [8] used it to improve the target recognition of the underwater environment. In the field of geography, Zhouqu et al. [9] applied it to the study of desert, Tongling et al. [10] applied it to the study of snow, Lovell et al. applied it to the study of vegetation, and found that the model coefficient is closely related to the spacing height ratio of vegetation elements.

In order to solve the spectral difference caused by the change of orientation in outdoor fruit non-destructive testing, this paper applies the roujean model to quantitatively describe the $f_{00}$ and Orient parameters of pBRDF of southern Xinjiang fruit, which provides a reference for improving the accuracy of outdoor fruit non-destructive testing in different orientations. There are many kinds of fruits in southern Xinjiang, so the two most representative fruits, jujube and apricot are selected as experimental materials.

\section{Materials and Methods}

\subsection{Test Materials}

The jujube was collected by image- $\lambda$-n17e-n 3 enhanced near infrared hyperspectral camera produced by Sichuan Shuangli Hepu Technology Co., Ltd. in the 10th regiment of the first division of Alar city, Xinjiang Uygur Autonomous Region. 


\subsection{Test Method}

108 jujubes of similar size and color were employed in the experiment. The dust on the surface was wiped with a soft cloth and labeled on the back in order. The standard diffuse reflectors were placed on the inner test stand, and the jujube was placed on the left and right test stands in order. The hyperspectral camera was installed and debugged at $1.5 \mathrm{~m}$ in front of the test stand, and the distance angle, detection altitude angle and detection azimuth angle from the camera to the diffuse reflector and jujube were recorded.

The test started at 13:00 Beijing time, the hyperspectral images of jujube and standard diffuse reflectors on the test stand were scanned under non polarization, $0^{\circ}$ polarization, $45^{\circ}$ polarization, $90^{\circ}$ polarization and $135^{\circ}$ polarization, respectively, and the solar altitude angle and solar azimuth angle were recorded. Then move the detector clockwise to $30^{\circ}$ and $45^{\circ}$ and repeat the above operation.

After the test, sample selection is performed. Figure 1 shows the nonpolarized hyperspectral image with the detector azimuth of $0^{\circ}$ and four samples with different positions in the fourth row, labeled as No.1-4; then 12 samples with different phase angles of No.5-8 and No.9-12 are selected from the images with detector azimuth of $30^{\circ}$ and $45^{\circ}$ and randomly selected according to the ratio of $3: 1$, of which nine are correction sets and three are prediction sets.

The test method of apricot is similar to above.

\section{3. pBRDF}

Polarized bidirectional reflectance distribution function (pBRDF) can characterize the reflection and scattering characteristics of materials. It is a fundamental optical parameter, which describes the distribution of reflected energy in the upper hemisphere after a light wave in an incident direction is reflected by the target surface. It is determined by surface roughness, dielectric constant, radiation wavelength, polarization and other factors, and its geometric relationship is illustrated in Figure 2.

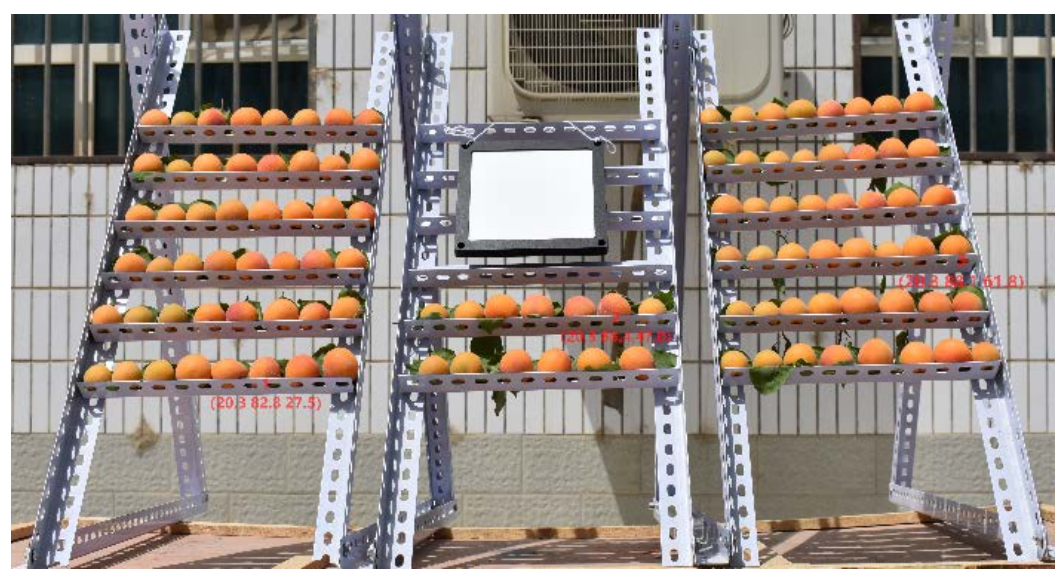

Figure 1. Sample selection diagram. 


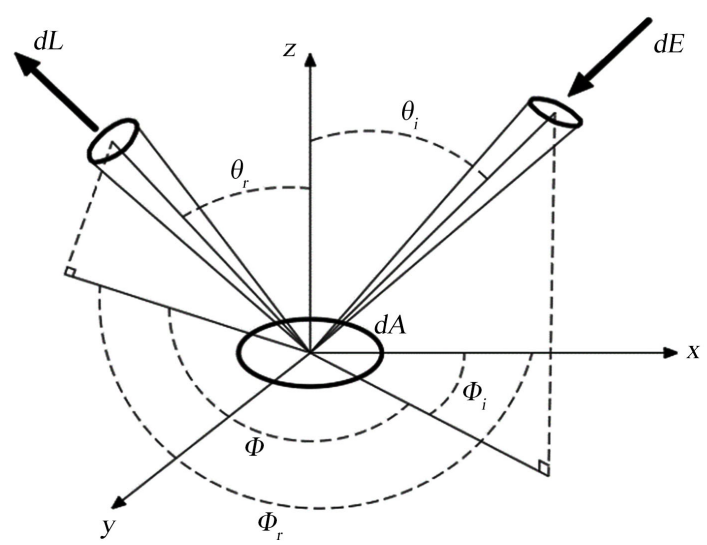

Figure 2. Geometrical relationship of pBRDF.

Note: the small surface element is $\mathrm{d} A$, the direction of incident light source is $\left(\theta_{i}, \Phi_{i}\right)$, and the observation direction of the detector is $\left(\theta_{r} \Phi_{r}\right)$. The subscripts $i$ and $r$ represent incident and reflection respectively, $\theta$ and $\Phi$ represent the zenith angle $\left({ }^{\circ}\right)$ and azimuth angle $\left({ }^{\circ}\right)$ respectively, and $Z$ represent average normal direction of the rough surface. $\mathrm{d} E$ is the irradiance in the direction of incident light source, and $\mathrm{d} L$ is the irradiance in the direction of reflection.

pBRDF is defined as the ratio of the radiance $\mathrm{d} L_{r}\left(\theta_{i}, \phi_{i}, \theta_{r}, \phi_{r}\right)$ reflected along the reflection direction of the irradiance $\mathrm{d} E_{i}\left(\theta_{i}, \phi_{i}\right)$ incident on the surface to be measured along the incident direction. The formula is as following:

$$
f_{r}\left(\theta_{i}, \theta_{i}, \phi, \lambda\right)=\frac{\mathrm{d} L_{r}\left(\theta_{i}, \theta_{r}, \phi\right)}{\mathrm{d} E_{i}\left(\theta_{i}, \phi_{i}\right)} .
$$

Note: $\lambda$ is the wavelength $(\mathrm{nm}) ; \theta=\theta_{r}-\theta_{i}$ The radiance $\left(L_{r}\right)$ is defined as the radiation flux $\left(\mathrm{W} /\left(\mathrm{m}^{2} \cdot \mathrm{sr}\right)\right)$ per unit area and solid angle along the radiation direction, while the irradiance $E_{i}$ is defined as the radiation flux $\left(\mathrm{W} / \mathrm{m}^{2}\right)$ per unit area, so the unit of $\mathrm{pBRDF}$ is $\mathrm{sr}^{-1}$.

\subsection{Measurement Principle of pBRDF}

In order to obtain the polarized BRDF data, it is necessary to design the test scheme through the polarized BRDF remote sensing radiation control equation, and determine the $F_{00}, F_{10}$, and $F_{20}$ elements of the BRDF matrix $\left(f_{00}, f_{10}\right.$, and $f_{20}$ are all PBRDF parameters, which can be used to calculate DOLP). The radiative transfer equation of $\mathrm{pBRDF}$ is as follows:

$$
\left[\begin{array}{l}
f_{00} \\
f_{10} \\
f_{20}
\end{array}\right]=\frac{\rho}{\pi\left(I_{s_{-} \text {ref } 90}+I_{s_{-} \text {ref } 0}\right)} \times\left[\begin{array}{c}
\left(I_{s_{-} 0}+I_{s_{-} 45}+I_{s_{-} 90}+I_{s_{-} 135}\right) / 2 \\
I_{s_{-} 0}-I_{s_{-} 90} \\
I_{s_{-} 45}-I_{s_{-} 135}
\end{array}\right] .
$$

Orient is an important parameter of pBRDF and plays an important role in polarization detection target recognition. The formula of Orient is as follows:

$$
\text { Orient }=\frac{1}{2} \arctan \left(\frac{f_{20}}{f_{10}}\right)
$$




\subsection{Roujean Model}

Roujean model has great advantages in describing pBRDF characteristics of vegetation, which is mainly represented by weighted linear combination of three cores:

1) Isotropic scattering function;

2) Geometric scattering function;

3) Volume scattering function.

$$
f_{00}=k_{0}+k_{1} f_{1}\left(\theta_{i}, \theta_{r}, \phi, \lambda\right)+k_{2} f_{2}\left(\theta_{i}, \theta_{r}, \phi, \lambda\right)
$$

Note: $f_{00}$ is the pBRDF value of scalar, and $f_{1}$ function is obtained by considering the geometric protuberance structure, which can be expressed as:

$$
\begin{aligned}
f_{1}\left(\theta_{i}, \theta_{r}, \phi, \lambda\right)= & \frac{1}{2 \pi}[(\pi-\phi) \cos \phi+\sin \phi] \tan \theta_{i} \tan \theta_{r} \\
& -\frac{1}{\pi}\left(\tan \theta_{i}+\tan \theta_{r}+\sqrt{\tan ^{2} \theta_{i}+\tan ^{2} \theta_{r}-2 \tan \theta_{i} \tan \theta_{r} \cos \phi}\right)
\end{aligned}
$$

The $f_{2}$ function is derived from a simple radiation model and can be expressed as:

$$
f_{2}\left(\theta_{i}, \theta_{r}, \phi, \lambda\right)=\frac{2}{3 \pi} \cdot \frac{(\pi-2 \xi) \cos \xi+2 \sin \xi}{\cos \theta_{i}+\cos \theta_{r}}-\frac{1}{3}
$$

Note: $\xi$ is the phase angle (the angle between the incident direction and the receiving direction), which can be expressed as:

$$
\cos \xi=\cos \theta_{i} \cos \theta_{r}+\sin \theta_{i} \sin \theta_{r} \cos \phi
$$

The parameters $k_{0}, k_{1}$ and $k_{2}$ are the weight of the corresponding scattering functions.

\subsection{Model Inversion}

In this experiment, firstly, orient was calculated from polarized hyperspectral data, and then the parameters $k_{0}, k_{1}, k_{2}$ of Roujean model were fitted by the least square method. Furthermore, the value of the Orient function in multi band with arbitrary incident and receiving directions is expected. The test data can be expressed as:

$$
F_{00}=K F
$$

where $F_{00}$ is a $254 \times 9$ orient data matrix, $K$ is a $254 \times 3$ roujean model parameter matrix, $K=\left[k_{0}, k_{1}, k_{2}\right]$, and $F$ is a $3 \times 9$ scattering function matrix, the $i$-th column represents the $i$-th measurement column vector $\left[\begin{array}{lll}1 & f_{1}^{(i)} & f_{2}^{(i)}\end{array}\right]^{\mathrm{T}}$, and $N$ represents the measurement times 9 . namely:

$$
F=\left[\begin{array}{ccc}
1 & \cdots & 1 \\
f_{1}^{(1)} & \cdots & f_{1}^{(n)} \\
f_{2}^{(1)} & \cdots & f_{2}^{(n)}
\end{array}\right]
$$

So the least square solution of $K$ is:

$$
K=F_{00} F^{\#}
$$


Note: $F$ is the pseudo inverse of $F^{*}, F^{\#}=F^{\mathrm{T}}\left(F F^{\mathrm{T}}\right)^{-1}$.

In this way, we can determine the Orient function value of the jujube in the southern Xinjiang, and the Roujean model parameters $k_{0}, k_{1}$ and $k_{2}$ in the multi band, and then predict the Orient function value in any incident and receiving directions. The parameter inversion process of the reflectance function of jujube in southern Xinjiang is the same as that of roujean model in multi band.

\section{Test Results and Analysis}

Table 1 and Table 2 show the comparison of experimental and predicted value of $f_{00}$ component of jujube and the parameter values of roujean model, respectively.

The results show that the average model errors of Roujean model for the prediction of jujube $f_{00}$ are $3.80 \%, 4.17 \%, 6.40 \% ; R^{2}$ are $0.9982,0.9963,0.9912$. Roujean model can be utilized to predict jujube $f_{00}$ very well. Figure 3 shows the comparison of the spectral curves of the experimental and predicted $f_{00}$ values of jujube.

Table 1. Comparison of experimental and predicted values of $f_{00}$ component in jujube.

\begin{tabular}{|c|c|c|c|}
\hline Wavelength/nm & Experimental value/sr ${ }^{-1}$ & Predicted value/sr ${ }^{-1}$ & Error $/ \%$ \\
\hline \multicolumn{4}{|c|}{ (a) $\theta_{\mathrm{r}}=54.2^{\circ}, \theta_{\mathrm{r}}=90^{\circ}, \Phi=51.9^{\circ}$} \\
\hline 1024 & 0.861344 & 0.858511385 & $0.33 \%$ \\
\hline 1124 & 0.586956 & 0.583145772 & $0.65 \%$ \\
\hline 1224 & 0.797584 & 0.7855544 & $1.51 \%$ \\
\hline 1324 & 0.373254 & 0.362944351 & $2.76 \%$ \\
\hline 1424 & 0.303363 & 0.289830387 & $4.46 \%$ \\
\hline 1524 & 0.285518 & 0.258873995 & $9.33 \%$ \\
\hline 1624 & 0.307102 & 0.283805326 & $7.59 \%$ \\
\hline \multicolumn{4}{|c|}{ (b) $\theta_{i}=52.2^{\circ}, \theta_{r}=90^{\circ}, \Phi=59.7^{\circ}$} \\
\hline 1024 & 0.882821 & 0.947324982 & $7.31 \%$ \\
\hline 1124 & 0.547986 & 0.58673964 & $7.07 \%$ \\
\hline 1224 & 0.856519 & 0.881795093 & $2.95 \%$ \\
\hline 1324 & 0.408383 & 0.422215333 & $3.39 \%$ \\
\hline 1424 & 0.284671 & 0.297303136 & $4.44 \%$ \\
\hline 1524 & 0.314227 & 0.319493384 & $1.68 \%$ \\
\hline 1624 & 0.339739 & 0.331650508 & $2.38 \%$ \\
\hline \multicolumn{4}{|c|}{ (c) $\theta_{i}=49.2^{\circ}, \theta_{r}=90^{\circ}, \Phi=87.8^{\circ}$} \\
\hline 1024 & 0.946735 & 0.935597561 & $1.18 \%$ \\
\hline 1124 & 0.555469 & 0.552130307 & $0.60 \%$ \\
\hline 1224 & 0.91835 & 0.868304603 & $5.45 \%$ \\
\hline 1324 & 0.46931 & 0.423480863 & $9.77 \%$ \\
\hline 1424 & 0.306292 & 0.27864765 & $9.03 \%$ \\
\hline 1524 & 0.402469 & 0.375908428 & $6.59 \%$ \\
\hline 1624 & 0.408904 & 0.358874548 & $12.25 \%$ \\
\hline
\end{tabular}


Table 2. Parameter values of roujean model.

\begin{tabular}{cccc}
\hline Wavelength/nm & $k_{0}$ & $k_{1}$ & $k_{2}$ \\
\hline 1024 & 2.214930804 & $7.47 \mathrm{E}-17$ & -2.343648741 \\
1124 & 1.019844474 & $3.28 \mathrm{E}-17$ & -0.731089566 \\
1224 & 2.264156257 & $8.16 \mathrm{E}-17$ & -2.554220613 \\
1324 & 1.166472205 & $4.19 \mathrm{E}-17$ & -1.39459688 \\
1424 & 0.601590897 & $2.17 \mathrm{E}-17$ & -0.526529391 \\
1524 & 1.023404717 & $3.84 \mathrm{E}-17$ & -1.330876363 \\
1624 & 0.863058267 & $2.84 \mathrm{E}-17$ & -1.010146741 \\
\hline
\end{tabular}

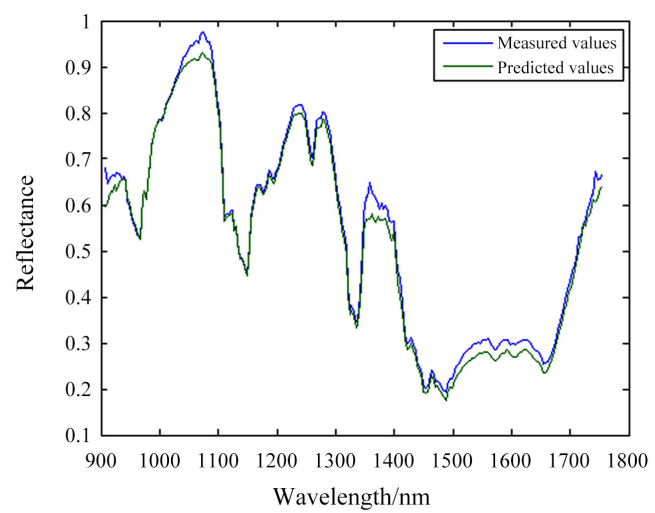

(a)

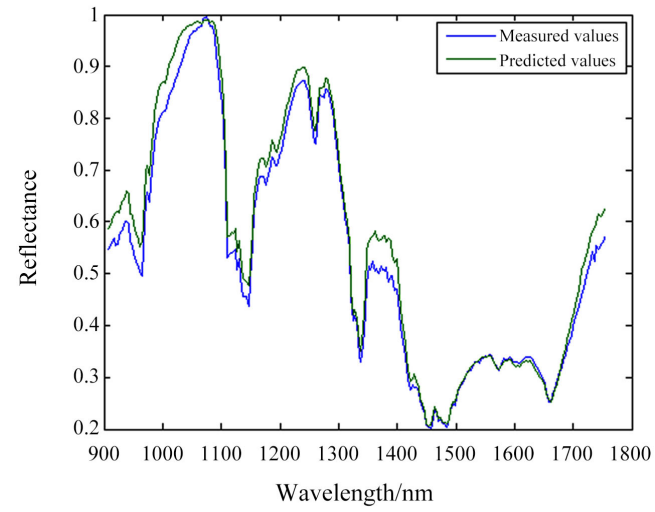

(b)

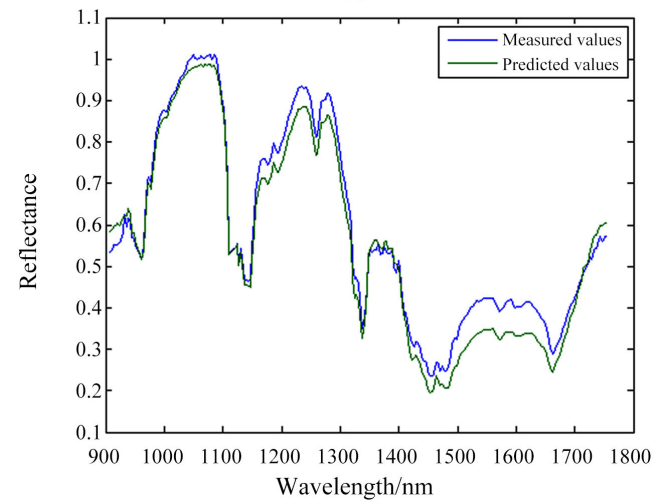

(c)

Figure 3. Comparison of the spectral curves of the test value and the predicted value of the Reflectance component of winter. (a) $\theta_{i}=54.2^{\circ}, \theta_{r}=90^{\circ}, \Phi=$ $51.9^{\circ}$; (b) $\theta_{i}=54.2^{\circ}, \theta_{r}=90^{\circ}, \Phi=40.9^{\circ}$; (c) $\theta_{i}=49.2^{\circ}, \theta_{r}=90^{\circ}, \Phi=96.3^{\circ}$. 
Table 3 and Table 4 show the comparison between the experimental and predicted values of jujube Orient and the parameter values of roujean model.

According to the experimental results, average model errors of roujean model for jujube orient were $6.23 \%, 3.32 \%$ and $8.05 \%$, and the $R^{2}$ were $0.9056,0.9223$ and 0.9260 , respectively. It can be seen that roujean model can better predict jujube Orient. Figure 4 shows the comparison of spectral curves between the experimental and predicted $f_{00}$ values of jujube.
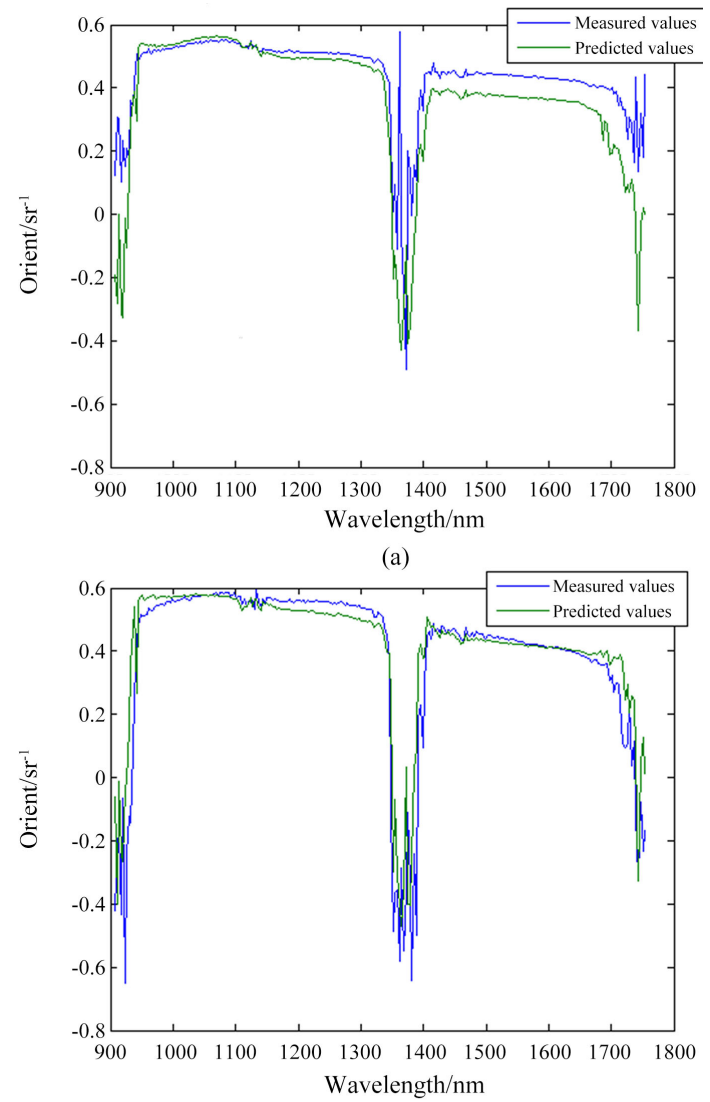

(b)

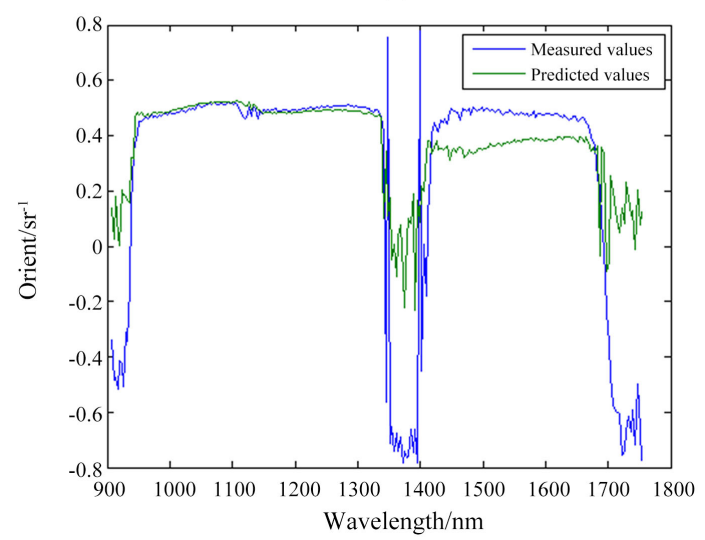

(c)

Figure 4. Comparison of the spectral curves of the test value and the predicted value of the Orient component of jujube. (a) $\theta_{i}=54.2^{\circ}, \theta_{r}=90^{\circ}, \Phi=$ $51.9^{\circ}$; (b) $\theta_{i}=54.2^{\circ}, \theta_{r}=90^{\circ}, \Phi=40.9^{\circ}$; (c) $\theta_{i}=49.2^{\circ}, \theta_{r}=90^{\circ}, \Phi=96.3^{\circ}$. 
Table 3. Comparison of experimental and predicted values of Orient component in jujube.

(a) $\theta_{i}=54.2^{\circ}, \theta_{r}=90^{\circ}, \Phi=51.9^{\circ}$

\begin{tabular}{|c|c|c|c|}
\hline Wavelength/nm & Experimental value/sr ${ }^{-1}$ & Predicted value/ $/ \mathrm{sr}^{-1}$ & Error $/ \%$ \\
\hline 1024 & 0.541189 & 0.546841512 & $1.04 \%$ \\
\hline 1124 & 0.549404 & 0.544266263 & $0.94 \%$ \\
\hline 1224 & 0.513433 & 0.49283233 & $4.01 \%$ \\
\hline 1324 & 0.485981 & 0.455994278 & $6.17 \%$ \\
\hline 1424 & 0.429555 & 0.376529352 & $12.34 \%$ \\
\hline 1524 & 0.4415 & 0.394986254 & $10.55 \%$ \\
\hline 1624 & 0.425466 & 0.38912603 & $8.53 \%$ \\
\hline \multicolumn{4}{|c|}{ (b) $\theta_{i}=54.2^{\circ}, \theta_{r}=90^{\circ}, \Phi=40.9^{\circ}$} \\
\hline 1024 & 0.562787 & 0.574227394 & $2.03 \%$ \\
\hline 1124 & 0.552012 & 0.570903122 & $3.42 \%$ \\
\hline 1224 & 0.558895 & 0.52370727 & $6.30 \%$ \\
\hline 1324 & 0.509832 & 0.484988318 & $4.87 \%$ \\
\hline 1424 & 0.457398 & 0.443700813 & $2.99 \%$ \\
\hline 1524 & 0.442066 & 0.426678165 & $3.48 \%$ \\
\hline 1624 & 0.405154 & 0.405772607 & $0.15 \%$ \\
\hline \multicolumn{4}{|c|}{ (c) $\theta_{i}=49.2^{\circ}, \theta_{r}=90^{\circ}, \Phi=96.3^{\circ}$} \\
\hline 1024 & 0.485134 & 0.504831738 & $4.06 \%$ \\
\hline 1124 & 0.479922 & 0.507027682 & $5.65 \%$ \\
\hline 1224 & 0.503343 & 0.489291979 & $2.79 \%$ \\
\hline 1324 & 0.486239 & 0.474720778 & $2.37 \%$ \\
\hline 1424 & 0.423246 & 0.379880553 & $10.25 \%$ \\
\hline 1524 & 0.494161 & 0.420065487 & $14.99 \%$ \\
\hline 1624 & 0.471158 & 0.394506385 & $16.27 \%$ \\
\hline
\end{tabular}

Table 4. Parameter values of roujean model.

\begin{tabular}{cccc}
\hline Wavelength/nm & $k_{0}$ & $k_{1}$ & $k_{2}$ \\
\hline 1024 & 0.280116743 & $-6.98 \mathrm{E}-18$ & 0.532741929 \\
1124 & 0.228574321 & $-1.05 \mathrm{E}-17$ & 0.613671779 \\
1224 & -0.488539378 & $-5.31 \mathrm{E}-17$ & 1.75598987 \\
1324 & -0.80810515 & $-7.26 \mathrm{E}-17$ & 2.230403147 \\
1424 & -1.930232344 & $-1.27 \mathrm{E}-16$ & 4.111760407 \\
1524 & -1.283696131 & $-8.99 \mathrm{E}-17$ & 2.966479767 \\
1624 & -1.756185047 & $-1.22 \mathrm{E}-16$ & 3.726832254 \\
\hline
\end{tabular}


Table 5 and Table 6 are the comparison of test value and predicted value of $f_{00}$ of apricot and the parameter value of roujean model.

According to the experimental results, average model errors of roujean model for predicting $f_{00}$ of apricot were $3.40 \%, 4.82 \%$ and $5.19 \%$, and the $R^{2}$ were 0.9862 , 0.9823 and 0.9792 respectively. Roujean model can well predict $f_{00}$ of apricot. Figure 5 shows the comparison of spectral curves of $f_{00}$ test value and the predicted value of apricot.

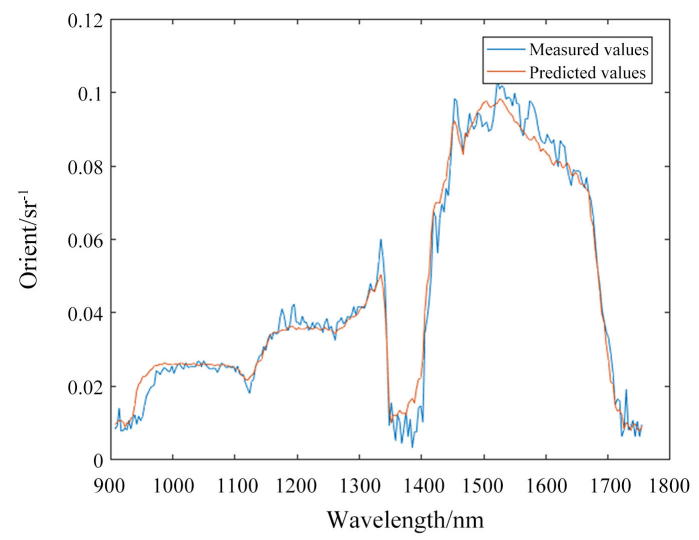

(a)

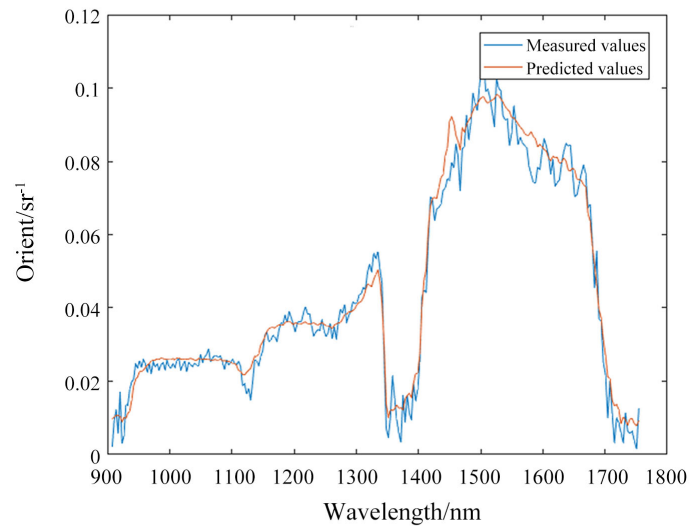

(b)

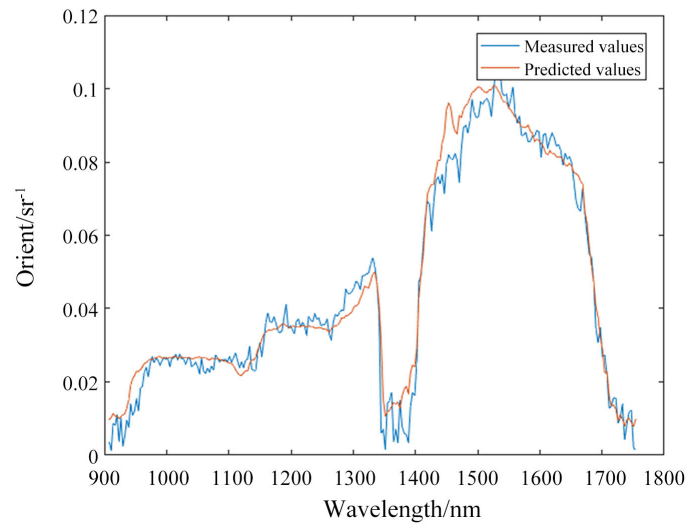

(c)

Figure 5. Comparison of the spectral curves of the test value and the predicted value of the f00 component of apricot. (a) $\theta_{i}=20.3^{\circ}, \theta_{r}=85.4^{\circ}, \Phi=$ $43.6^{\circ}$; (b) $\theta_{i}=20.3^{\circ}, \theta_{r}=85.4^{\circ}, \Phi=44.7^{\circ}$; (c) $\theta_{i}=20.3^{\circ}, \theta_{r}=82.7^{\circ}, \Phi=41.7^{\circ}$. 
Table 5. Comparison of test value and the predicted value of $f_{00}$ component in apricot.

\begin{tabular}{|c|c|c|c|}
\hline \multicolumn{4}{|c|}{ (a) $\theta_{i}=20.3^{\circ}, \theta_{r}=85.4^{\circ}, \Phi=43.6^{\circ}$} \\
\hline Wavelength/nm & Experimental value $/ \mathrm{sr}^{-1}$ & Predicted value/sr ${ }^{-1}$ & Error/\% \\
\hline 1024 & 0.025249 & 0.026126 & $3.57 \%$ \\
\hline 1124 & 0.017103 & 0.017423 & $0.18 \%$ \\
\hline 1224 & 0.041458 & 0.039617 & $4.35 \%$ \\
\hline 1324 & 0.046181 & 0.045676 & $1.09 \%$ \\
\hline 1424 & 0.066151 & 0.069926 & $5.71 \%$ \\
\hline 1524 & 0.100965 & 0.098186 & $2.78 \%$ \\
\hline 1624 & 0.096948 & 0.091062 & $6.13 \%$ \\
\hline \multicolumn{4}{|c|}{ (b) $\theta_{i}=17.9^{\circ}, \theta_{r}=85.5^{\circ}, \Phi=40.8^{\circ}$} \\
\hline 1024 & 0.02551 & 0.026115 & $2.37 \%$ \\
\hline 1124 & 0.017453 & 0.018408 & $5.75 \%$ \\
\hline 1224 & 0.038447 & 0.035598 & $7.41 \%$ \\
\hline 1324 & 0.047696 & 0.045658 & $4.20 \%$ \\
\hline 1424 & 0.0695 & 0.069929 & $0.62 \%$ \\
\hline 1524 & 0.102518 & 0.098189 & $4.22 \%$ \\
\hline 1624 & 0.074242 & 0.081061 & $9.18 \%$ \\
\hline \multicolumn{4}{|c|}{ (c) $\theta_{i}=21.4^{\circ}, \theta_{r}=83.8^{\circ}, \Phi=48.9^{\circ}$} \\
\hline 1024 & 0.026238 & 0.026808 & $2.17 \%$ \\
\hline 1124 & 0.023725 & 0.02243 & $5.46 \%$ \\
\hline 1224 & 0.042651 & 0.038909 & $8.69 \%$ \\
\hline 1324 & 0.05972 & 0.055551 & $6.98 \%$ \\
\hline 1424 & 0.068411 & 0.072873 & $6.52 \%$ \\
\hline 1524 & 0.098999 & 0.100781 & $1.80 \%$ \\
\hline 1624 & 0.086514 & 0.082427 & $4.72 \%$ \\
\hline
\end{tabular}

Table 6. Parameter values of roujean model.

\begin{tabular}{cccc}
\hline Wavelength/nm & $k_{0}$ & $k_{1}$ & $k_{2}$ \\
1024 & 0.768387129 & -0.000465164 & 0.001381702 \\
1124 & 0.672129504 & -0.000358553 & 0.001445897 \\
1224 & 0.693408698 & -0.00053167 & 0.001264161 \\
1324 & 0.422589673 & -0.000379925 & 0.000798369 \\
1424 & 0.336883244 & -0.00054806 & 0.000466328 \\
1524 & 0.301925877 & -0.000535819 & 0.000261745 \\
1624 & 0.327140747 & -0.000346854 & 0.000425857 \\
\hline
\end{tabular}


Table 7 and Table 8 show the comparison between the experimental value and the predicted value and the parameter value of roujean model.

According to the experimental results, average model errors of roujean model for the prediction value of apricot orient were $7.19 \%, 9.28 \%$ and $9.47 \%$, and the $R^{2}$ were $0.9382,0.8947$ and 0.8849 , respectively. Roujean model can predict the $f_{00}$ of apricot better. Figure 6 shows the comparison of spectral curves of $f_{00}$ test value and the predicted value of apricot.

Table 9 compares the intensity component and polarization phase angle of roujean model in quantitative description of jujube and Baixing in southern Xinjiang.

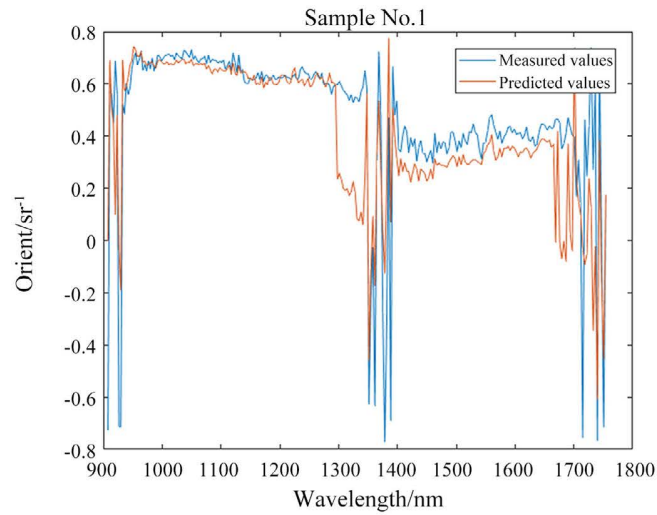

(a)

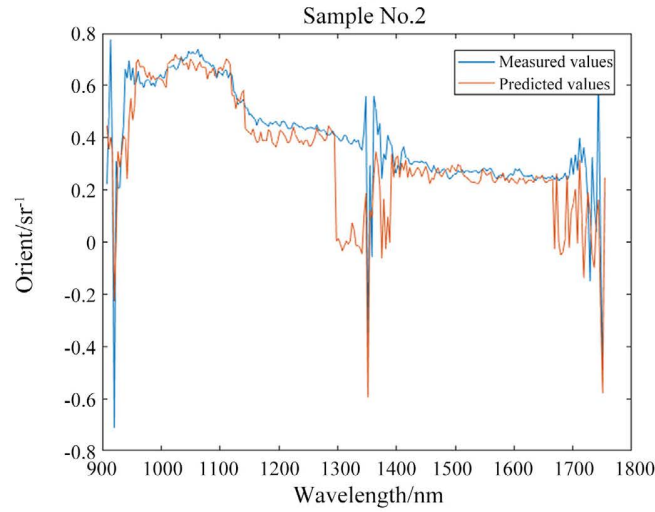

(b)

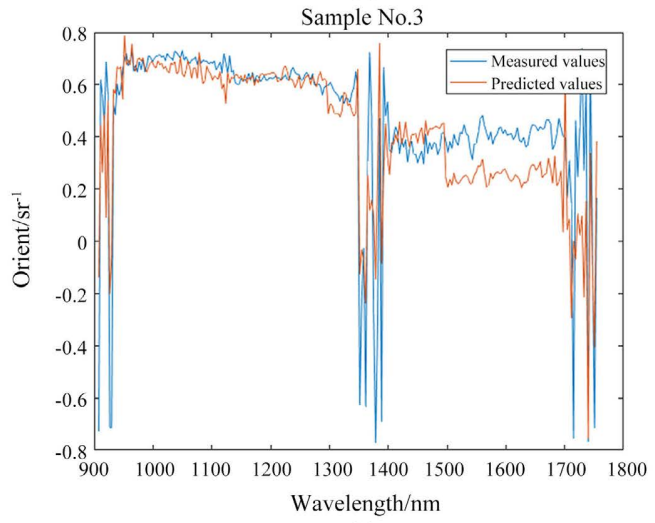

Figure 6. Comparison of the spectral curves of the test value and the predicted value of the Orient component of apricot. (a) $\theta_{i}=20.3^{\circ}, \theta_{r}=85.4^{\circ}, \Phi=43.6^{\circ}$; (b) $\theta_{i}=20.3^{\circ}, \theta_{r}=85.4^{\circ}, \Phi=44.7^{\circ}$; (c) $\theta_{i}=20.3^{\circ}, \theta_{r}=82.7^{\circ}, \Phi=41.7^{\circ}$. 
Table 7. Comparison of test value and the predicted value of Orient component in apricot.

(a) $\theta_{i}=20.3^{\circ}, \theta_{r}=85.4^{\circ}, \Phi=43.6^{\circ}$

\begin{tabular}{|c|c|c|c|}
\hline Wavelength/nm & Experimental value/sr ${ }^{-1}$ & Predicted value/sr ${ }^{-1}$ & Error/\% \\
\hline 1024 & 0.719129 & 0.692097434 & $3.76 \%$ \\
\hline 1124 & 0.683017 & 0.642952323 & $5.87 \%$ \\
\hline 1224 & 0.637972 & 0.661506272 & $3.69 \%$ \\
\hline 1324 & 0.526909 & 0.505321971 & $4.10 \%$ \\
\hline 1424 & 0.361483 & 0.320295342 & $11.39 \%$ \\
\hline 1524 & 0.413654 & 0.373237722 & $9.77 \%$ \\
\hline 1624 & 0.385385 & 0.340028096 & $11.77 \%$ \\
\hline \multicolumn{4}{|c|}{ (b) $\theta_{i}=17.9^{\circ}, \theta_{r}=85.5^{\circ}, \Phi=40.8^{\circ}$} \\
\hline 1024 & 0.662113 & 0.719186524 & $8.62 \%$ \\
\hline 1124 & 0.574294 & 0.561432584 & $2.24 \%$ \\
\hline 1224 & 0.45858 & 0.401245358 & $12.50 \%$ \\
\hline 1324 & 0.37704 & 0.343482743 & $8.90 \%$ \\
\hline 1424 & 0.297907 & 0.272620044 & $8.49 \%$ \\
\hline 1524 & 0.266751 & 0.23262322 & $12.79 \%$ \\
\hline 1624 & 0.253066 & 0.224206016 & $11.40 \%$ \\
\hline \multicolumn{4}{|c|}{ (c) $\theta_{1}=21.4^{\circ}, \theta_{r}=83.8^{\circ}, \Phi=48.9^{\circ}$} \\
\hline 1024 & 0.719129 & 0.652754839 & $9.23 \%$ \\
\hline 1124 & 0.683017 & 0.627267503 & $8.16 \%$ \\
\hline 1224 & 0.637972 & 0.573103103 & $10.17 \%$ \\
\hline 1324 & 0.526909 & 0.571239607 & $8.41 \%$ \\
\hline 1424 & 0.361483 & 0.399360907 & $10.48 \%$ \\
\hline 1524 & 0.413654 & 0.442053351 & $6.87 \%$ \\
\hline 1624 & 0.385385 & 0.335484195 & $12.95 \%$ \\
\hline
\end{tabular}

Table 8. Parameter values of roujean model.

\begin{tabular}{cccc}
\hline Wavelength/nm & $k_{0}$ & $k_{1}$ & $k_{2}$ \\
1024 & 0.280515 & -0.01057 & 0.068588 \\
1124 & 0.212257 & -0.01772 & 0.069441 \\
1224 & 0.278587 & -0.00399 & 0.065854 \\
1324 & 0.162159 & 0.00075 & 0.011355 \\
1424 & 0.216234 & 0.002202 & 0.008508 \\
1524 & 0.219979 & 0.001693 & 0.015214 \\
1624 & 0.19941 & 0.002855 & 0.025694 \\
\hline
\end{tabular}


Table 9. Comparison of $f_{00}$ and Orient models between jujube and apricot in southern Xinjiang.

\begin{tabular}{cccccc}
\hline Research objectives & Sample number & $\zeta\left(^{\circ}\right)$ & $R^{2}$ & RMSEP & Error/\% \\
\hline Jujube $f_{00}$ & 3 & 52.19 & 0.9982 & 0.0224 & $3.80 \%$ \\
\hline Jujube Orient & 11 & 66.51 & 0.9963 & 0.0346 & $4.17 \%$ \\
& 3 & 88.33 & 0.9912 & 0.0437 & $6.40 \%$ \\
\hline Apricot $f_{00}$ & 11 & 52.19 & 0.9056 & 0.1426 & $6.23 \%$ \\
& 2 & 66.51 & 0.9223 & 0.1126 & $3.32 \%$ \\
& 4 & 88.33 & 0.926 & 0.3055 & $8.05 \%$ \\
\hline Apricot Orient & 8 & 70.99 & 0.9862 & 0.044 & $3.40 \%$ \\
& 2 & 71.28 & 0.9823 & 0.0140 & $4.82 \%$ \\
& 4 & 67.91 & 0.9792 & 0.0761 & $5.19 \%$ \\
\hline
\end{tabular}

It can be seen from Table 8 that roujean model can well predict $f_{00}$ of jujube and Baixing, and can better predict Orient, and the overall prediction effect of jujube is better than that of Baixing.

\section{Conclusions}

1) The experimental results show that the maximum model error and minimum model error are $6.40 \%$ and $3.32 \%$ when describing winter jujube $f_{00}$ by Roujean model, $8.05 \%$ and $3.71 \%$ respectively when describing winter jujube Orient. The maximum model error and minimum model error are 5.19\% and $3.40 \%$ when describing White apricot $f_{00}$ by Roujean model, $9.47 \%$ and $7.19 \%$ respectively when describing White apricot Orient. In conclusion, The Roujean model can effectively describe the $f_{00}$ and Orient parameters of southern Xinjiang fruit, so as to solve the difference caused by the orientation change during outdoor non-destructive testing, and provide a reference for improving the accuracy of outdoor non-destructive testing.

2) Due to the limited test conditions, the incident zenith angle, detection zenith angle, and relative azimuth angle required for the test are calculated after measurement, and there are errors in their accuracy. Follow-up outdoor tests can improve the accuracy and general adaptability of the model by using high-precision laser rangefinders, adding more angle tests, and increasing the number and types of fruits.

\section{Acknowledgements}

This paper and the study are funded by: Fund Project 1: National Natural Science Foundation of China "Study on multi-scale hyperspectral polarization 
quantitative remote sensing model of jujube in southern Xinjiang" (Project No. 11964030); Fund Project 2: National Natural Science Foundation of China "Study on polarization remote sensing mechanism of jujube quality in southern Xinjiang Based on polarization dichroism" (Project No. 11164023).

\section{Conflicts of Interest}

The authors declare no conflicts of interest regarding the publication of this paper.

\section{References}

[1] Yang, P.Y. and Zhang, Z.M.M. (2020) Bidirectional Reflection of Semitransparent Polytetrafluoroethylene (PTFE) Sheets on a Silver Film. International Journal of Heat and Mass Transfer, 148, Article No. 118992.

https://doi.org/10.1016/j.ijheatmasstransfer.2019.118992

[2] Shinichi, I.,Masanori, M. and Norimichi, T. (2020) Mathematical Model of Paper Surface Topography by Perlin Noise Derived from Optical Reflection Characteristics. Japan Tappi Journal, 73, 2022-1029.

[3] , Falster, L.V. Doest, M.B., Ribo, M.M., Eiriksson, E.R., Pedersen, D.B. andFrisvad, J.R. (2020) Microstructure Control in 3D Printing with Digital Light Processing. Computer Graphics Forum, 39, 347-359. https://doi.org/10.1111/cgf.13807

[4] Zhang, Y.-L., Wang, Y.-M., Huang, A.-P., et al. (2020) Study on Reflection Characteristics of Underwater Target and Laser Echo Power. Optoelectronics Letters, 16, 137-142. https://doi.org/10.1007/s11801-020-9104-3

[5] Kristan, P.G. and Melvin, F. (2012) Remote Detection of Buried Land-Mines and IEDs Using LWIR Polarimetric Imaging. Optical Society of America, 20, 22344-22359. https://doi.org/10.1364/OE.20.022344

[6] Yeom, J.-M., Roujean, J.-L., Han, K.-S., et al. (2020) Thin Cloud Detection over Land Using Background Surface Reflectance Based on the pBRDF Model Applied to Geostationary Ocean Color Imager (GOCI) Satellite Data Sets. Remote Sensing of Environment, 239, 111610-111623. https://doi.org/10.1016/j.rse.2019.111610

[7] Farhad, M.M., Morakot, K., Larry, L. et al. (2020) Radiometric Cross Calibration and Validation Using 4 Angle pBRDF Model between Landsat 8 and Sentinel 2A. Remote Sensing, 12, 806-826. https://doi.org/10.3390/rs12050806

[8] Aval, J., Alfalou, A. and Brosseau, C. (2019) Polarization and Hyperspectral Imaging Matter for Newly Emerging Perspectives in Optical Image Processing: Guest Editorial. Optical Society of America, 11, 10-14.

https://doi.org/10.1364/AOP.11.00ED10

[9] Zhou, Q., Tian, L.Q., Li, J., et al. (2020) Assessment of Bidirectional Reflectance Effects on Desert and Forest for Radiometric Cross-Calibration of Satellite Sensors. ISPRS Journal of Photogrammetry and Remote Sensing, 160, 733-749. https://doi.org/10.1016/j.isprsjprs.2019.12.007

[10] Wu, H.Y. and Tong, L. (2011) Modeling Visible and Near-Infrared Snow Surface Reflectance-Simulation and Validation. Chinese Optics Letters, 9, 83-85.

https://doi.org/10.3788/COL201109.102901 